\title{
Microembolic signal monitoring of TOAST-classified cerebral infarction patients
}

\author{
JIANDONG JIANG $^{1}$, YULONG JIANG ${ }^{1}$, SHOUQIN FENG ${ }^{2}$, DEJIN SUN ${ }^{1}$, AIXIA ZHUANG ${ }^{1}$, QINGHONG ZENG ${ }^{1}$, \\ YI ZHANG $^{3}$, HONGMEI HUANG ${ }^{4}$, HONGXIA NIE $^{1}$ and FANG ZHOU ${ }^{1}$
}

Departments of ${ }^{1}$ Neurology, ${ }^{2}$ Neurological Examination, ${ }^{3}$ Radiology and ${ }^{4}$ Ultrasound, Lianyungang Second Hospital, Lianyungang, Jiangsu 222006, P.R. China

Received October 19, 2012; Accepted June 19, 2013

DOI: $10.3892 / \mathrm{mmr} .2013 .1609$

\begin{abstract}
The aim of the present study was to investigate the effects of microembolic signals (MES) on post-stroke neurological deficits, stroke recurrence and survival in patients with acute cerebral infarction (CI). Patients with acute CI were enrolled consecutively and classified etiologically into the following 4 subtypes using the Trial of Org 10172 in Acute Stroke Treatment (TOAST) classification: i) Cardioembolism (CE); ii) large-artery atherosclerosis (LA); iii) small-vessel disease (SVD); and iv) stroke of other etiology, including other and undetermined etiologies. The MES of cerebral arteries were monitored by transcranial doppler (TCD), carotid atherosclerotic lesions were detected by color Doppler sonography and extracranial and intracranial magnetic resonance angiography were performed. Next, the severity of neurological deficits was evaluated using National Institutes of Health Stroke Scale (NIHSS) scores. Cases of stroke recurrence and post-stroke mortality were recorded. A total of 135 patients were recruited, including 33 with CE, 49 with LA, 24 with SVD and 29 with stroke of other etiology. A significant difference in the incidence of MES was identified between the 4 subtypes $(\mathrm{P}=0.025)$. The occurrence of positive MES was found to markedly correlate with a history of coronary heart disease $(\mathrm{P}<0.001)$ and antithrombotic treatment $(\mathrm{P}=0.045)$ and increased levels low density lipoprotein $(\mathrm{P}=0.036)$. Compared with patients with negative MES, no significant changes in NIHSS scores were found in patients with positive MES on days 1 and 7 following admission. The incidence of recurrent stoke and post-stroke mortality was elevated 3 months from the onset of CI. In conclusion, a significant difference in the occurrence of MES was identified between subtypes of patients with acute CI. The incidence of recurrent stroke and
\end{abstract}

Correspondence to: Dr Aixia Zhuang, Department of Neurology, Lianyungang Second Hospital, No. 41 Hailian Dong Road, Xinpu, Lianyungang, Jiangsu 222006, P.R. China

E-mail: zhuangaixiacn@126.com

Key words: acute cerebral infarction, TOAST classification, microembolic signal, transcranial doppler, stroke recurrence mortality was increased among patients with positive MES 3 months from onset.

\section{Introduction}

In 1990, Spencer et al (1) identified an abnormal signal from solid particles in patients with carotid endarterectomy using transcranial doppler (TCD) cerebral blood flow monitoring, proposing a definition of microembolic signals (MES). In 1995, a specialist consensus on the diagnostic criteria of MES was published in the journal Stroke (2). TCD microemboli detection is often used to identify and monitor patients with carotid stenosis, atrial fibrillation and artificial heart valve disease, as well as in patients who have undergone carotid intravascular surgery and interventional therapy $(3,4)$. A number of studies have revealed that microembolisms correlate with the severity of intracranial artery stenosis $(5,6)$, and as the acute stage of cerebral infarction (CI) progresses, the incidence of microembolism decreases (7). In patients with CI, the presence of microembolisms is associated with lesion number and characteristics in cranial magnetic resonance imaging, including diffusion-weighted imaging (MRI DWI) (8-10), whereby microemboli-positive patients exhibit a higher probability of CI (5,11-13). However, previous studies have mainly focused on atherosclerotic thrombotic CI, namely, large artery CI, and the correlation between CI subgroup and incidence of microemboli is seldom involved. In the the present study, CI patients were classified etiologically into subtypes using the Trial of Org 10172 in Acute Stroke Treatment (TOAST) classification method and TCD microemboli detection was performed on CI patients $48 \mathrm{~h}, 1$ week and 3 months following CI in order to determine the effect of CI microembolism in patients of various TOAST classifications on post-stroke nerve function deficit, stroke recurrence and mortality.

\section{Materials and methods}

Patients. Patients were recruited within $48 \mathrm{~h}$ of acute CI according to diagnostic criteria outlined previously (2). Patients were 18-80 years old. Informed consent was acquired from patients or their families. According to TOAST classification, cases were divided into the following 4 subtypes: i) cardioembolism (CE, patients with a clear cardiogenic basis disease 
and cardiogenic embolus); ii) large-artery atherosclerosis (LA, patients with CI of artery atherosclerosis); iii) small-vessel disease (SVD; patients with lacunar infarction); and iv) stroke of other etiology, including other and undetermined etiologies (i.e., acleistocardia, Moyamoya disease, carotid artery interlayer or other unknown agnogenic diseases). Following a study performed by DeGraba et al (14), individuals with a neurological deficit National Institutes of Health Stroke Scale (NIHSS) score of 0-6 and $>6$ points were included in the mild-moderate and severe groups, respectively.

Patients were excluded in cases where the segment distal to the stenosis lesion was insonated through the temporal window or when patients were unable to tolerate 30 min of microemboli monitoring and microemboli monitoring was not completed. In addition, patients with poor compliance were not included.

TCD microemboli monitoring. TCD microemboli detection was performed within $48 \mathrm{~h}$ of CI. In microemboli-positive patients, additional microemboli tests were performed at 1 week and 3 months following CI. A transcranial doppler ultrasound detector (Embo-Dop; DWL Elektronische Systeme $\mathrm{GmbH}$, Singen, Germany) was used to monitor microemboli. Extracranial and intracranial blood vessels were tested using 2 and $4 \mathrm{MHz}$ probes and the bilateral middle cerebral artery (MCA) was checked. Probes were fixed following exploration of the MCA in the temporal window of the lesion side and microemboli monitoring was performed in the MCA initial segment or narrow distal end (sampling depth, 48-58 mm; distance between two points, $\geq 6 \mathrm{~cm}$; sample volume, $6-12 \mathrm{~cm}$; MES relative threshold, $>5 \mathrm{~dB}$; and duration of microemboli monitoring, $30 \mathrm{~min}$ ). The signal was rechecked offline to eliminate the artifact and interference and the results were recorded using Professional TCD software VI 3.3 (TA-88, TEAC Corporation, Tokyo, Japan). Microemboli monitoring was performed by a system-trained professional and identified by a senior neurology physician. The microembolus was distinguished according to criteria outlined previously (2).

Carotid ultrasound detection. The Philips IU22 color ultrasound diagnostic apparatus with a 5-12 MHz probe (Philips, Eindhoven, Holland) was used to analyze the following parameters: Common carotid artery, artery bifurcation, internal carotid artery, intima thickness, lumen diameter, plaque and plaque size, shape and echo characteristics. This allowed the results to be divided into 3 groups as follows: i) No plaque; ii) stable plaque, including fibers and calcified hard plaques; and iii) unstable plaque, including soft plaques, ulcers and mixed plaques.

Cranial MRI DWI test. DWI was performed using a 1.5-T MRI (GE Healthcare, Pewaukee, WI, USA) with a standard head coil. A multishot spin-echo echo-planar imaging sequence was employed and diffusion-weighted b-values of 0.313 and $1.252 \mathrm{~s} / \mathrm{mm}^{2}$ were used to obtain 9 slices in each of 3 principal magnet directions. Patients with aggravation or recurrence of $\mathrm{CI}$ underwent a second MRI. Magnetic resonance angiogram (MRA) examinations were performed on head and neck arteries in all patients. Vascular stenosis was diagnosed by one positive result from TCD, carotid artery ultrasound or head-neck MRA (15).
Neural function defect evaluation. NIHSS grading was performed immediately and also 1 week following admission. Modified Rankin scale (mRS) grading was performed during outpatient follow-ups prior to discharge from hospital and 3 months following CI. Patients who did not attend outpatient appointments to seek medical advice were contacted by telephone for mRS evaluation and to record mortalities.

Stroke risk factors and general indicators. Patient information, including age, gender, history of smoking, drinking, hypertension, diabetes, stroke, coronary heart disease and atrial fibrillation, as well as levels of blood sugar, blood lipids, blood homocysteine, c-reactive protein, fibrinogen and glutamyl endopeptidase, plus general patient information, was collected.

Statistical methods. Statistical analysis was performed using the SPSS 11.5 software package (SPSS, Inc., Chicago, IL, USA). Chi-square tests were used to analyze nominal data, t-tests were used for comparison between two sets of data and ANOVA was used for comparison between several groups. Microemboli-related factors were analyzed using logistic regression analysis. $\mathrm{P}<0.05$ was considered to indicate a statistically significant difference.

\section{Results}

TOAST classification. Between January 2008 and October 2011, 1,000 inpatients with ischemic stroke were admitted to the Neurology Department of the Second People's Hospital of Lianyungang and 256 eligible patients were preliminarily selected. Among them, 44 patients refused carotid ultrasound or head MRI, 68 patients were found to exhibit closed temporal ultrasound windows via a TCD test, 6 microemboli-positive patients refused a second microemboli test leading to incomplete data and 3 patients lost in 3 months. Finally, 135 patients were divided into 4 research groups within $48 \mathrm{~h}$ following CI. Among them, there were 33 patients in the CE group, 49 patients in the LA group, 24 patients in the SVD group and 29 patients in the other etiology group (patients with other or unknown etiologies).

Etiology, risk factors and general information of TOAST classified subgroups. Patients were divided into groups according to etiology. Marked differences in patient history of hypertension and atrial fibrillation, as well as a number of other cerebrovascular etiology indexes were found between these groups, including blood sugar, c-reactive protein, total cholesterol, homocysteine levels and other indices; however, these differences were not statistically significant. Age, gender, platelet count, hematocrit and neurological deficit NIHSS scores were unchanged between the subtypes (Table I).

Etiology, stroke risk factors and general information of microemboli-positive and-negative patients following enrollment. Microemboli-positive and -negative patients were found to exhibit significant differences in history of coronary heart disease (OR, 5.862; 95\% CI, 2.008-17.114; $\mathrm{P}=0.000)$ and antithrombotic therapy (OR, 0.376; 95\% CI, 0.141-1.002; $\mathrm{P}=0.045$ ) and low density lipoprotein levels $(\mathrm{P}=0.036)$. By contrast, gender (OR, 0.850; 95\% CI, 0.311-2.321; $\mathrm{P}=0.750$ ), history 
Table I. Etiology, risk factors and general information of TOAST-classified subgroups.

\begin{tabular}{|c|c|c|c|c|c|}
\hline \multirow[b]{2}{*}{ Item } & \multicolumn{4}{|c|}{ TOAST typing and grouping } & \multirow[b]{2}{*}{ P-value } \\
\hline & $\begin{array}{l}\text { Cerebral embolism } \\
\qquad(\mathrm{n}=33)\end{array}$ & $\begin{array}{l}\text { Aorta disease } \\
\quad(n=49)\end{array}$ & $\begin{array}{l}\text { Arteriolopathy } \\
\qquad(\mathrm{n}=24)\end{array}$ & $\begin{array}{l}\text { Other } \\
(n=29)\end{array}$ & \\
\hline Gender male, n (\%) & $27(81.8)$ & $35(71.4)$ & $18(75.0)$ & $23(79.3)$ & 0.713 \\
\hline Average age, years & $66.91 \pm 11.16$ & $63.45 \pm 9.44$ & $65.08 \pm 11.11$ & $65.28 \pm 10.51$ & 0.530 \\
\hline \multicolumn{6}{|l|}{ Patient history, n (\%) } \\
\hline Apoplexy & $6(18.2)$ & $4(8.2)$ & $1(4.2)$ & $2(6.9)$ & 0.265 \\
\hline Atrial fibrillation & $28(84.8)$ & 0 & $1(4.2)$ & 0 & 0.000 \\
\hline Hypertension & $17(51.5)$ & $33(67.3)$ & $18(75.0)$ & 0 & 0.000 \\
\hline Diabetes & $5(15.2)$ & $18(36.7)$ & $5(20.8)$ & 0 & 0.001 \\
\hline Coronary heart & $5(15.2)$ & $8(16.3)$ & $4(16.7)$ & 0 & 0.147 \\
\hline Alcohol consumption & $9(27.3)$ & $19(38.8)$ & $9(37.5)$ & $4(13.8)$ & 0.105 \\
\hline Smoking & $11(33.3)$ & $21(42.9)$ & $10(41.7)$ & $5(17.2)$ & 0.118 \\
\hline Antithrombotic therapy & $15(51.5)$ & $22(51.5)$ & $10(51.5)$ & $4(51.5)$ & 0.028 \\
\hline Hematocrit (\%) & $40.11 \pm 5.45$ & $39.60 \pm 4.91$ & $41.80 \pm 5.10$ & $39.52 \pm 3.71$ & 0.284 \\
\hline Blood platelet count, x $10^{9}$ & $219.2 \pm 56.63$ & $224.8 \pm 66.2$ & $205.6 \pm 77.5$ & $195.7 \pm 42.7$ & 0.202 \\
\hline Fibrinogen, mg/l & $3.05 \pm 0.657$ & $2.84 \pm 0.779$ & $2.64 \pm 0.603$ & $2.74 \pm 0.661$ & 0.145 \\
\hline c-reactive protein, mmol/1 & $3.128 \pm 3.349$ & $3.239 \pm 3.364$ & $3.176 \pm 4.754$ & $2.993 \pm 3.394$ & 0.096 \\
\hline Blood glucose, mmol/1 & $6.259 \pm 3.277$ & $7.156 \pm 3.558$ & $6.271 \pm 1.797$ & $5.706 \pm 2.304$ & 0.098 \\
\hline Total cholesterol, mmol/l & $4.229 \pm 1.145$ & $4.749 \pm 1.013$ & $4.566 \pm 1.011$ & $4.196 \pm .804$ & 0.061 \\
\hline Triglyceride, mmol/1 & $1.342 \pm 0.713$ & $1.622 \pm 1.042$ & $1.458 \pm 1.175$ & $1.325 \pm .845$ & 0.512 \\
\hline Low density lipoprotein, mmol/1 & $2.603 \pm 0.849$ & $2.959 \pm 0.839$ & $2.554 \pm 0.692$ & $2.807 \pm 0.786$ & 0.123 \\
\hline High density lipoprotein, mmol/l & $1.069 \pm 0.247$ & $1.121 \pm 0.272$ & $1.075 \pm 0.269$ & $1.119 \pm 0.185$ & 0.741 \\
\hline Apolipoprotein A, mmol/l & $1.153 \pm 0.279$ & $1.173 \pm 0.229$ & $1.217 \pm 0.291$ & $1.141 \pm 0.218$ & 0.714 \\
\hline Apolipoprotein B, mmol/l & $1.109 \pm 0.923$ & $1.024 \pm 0.256$ & $1.133 \pm 1.413$ & $0.945 \pm 0.259$ & 0.786 \\
\hline Homocysteic acid, mmol/1 & $15.20 \pm 6.68$ & $18.30 \pm 12.96$ & $13.64 \pm 7.07$ & $14.88 \pm 7.19$ & 0.069 \\
\hline Glutamyltranspeptidase, U/1 & $35.18 \pm 20.33$ & $40.13 \pm 31.24$ & $35.18 \pm 20.02$ & $32.91 \pm 16.12$ & 0.592 \\
\hline Nerve function scores & $4.85 \pm 4.23$ & $5.00 \pm 3.47$ & $4.29 \pm 3.43$ & $4.59 \pm 3.43$ & 0.876 \\
\hline $\begin{array}{l}\text { NIHSS score grouping } \\
\text { (mild-moderate), n (\%) }\end{array}$ & $26(78.8)$ & $36(73.5)$ & $17(70.8)$ & $21(72.4)$ & 0.905 \\
\hline
\end{tabular}

TOAST, Trial of Org 10172 in Acute Stroke Treatment.

of hypertension (OR, 1.697; 95\% CI, 0.727-3.962; $\mathrm{P}=0.219)$, diabetes (OR, 2.194; 95\% CI, 0.861- 5.590; $\mathrm{P}=0.095)$, atrial fibrillation (OR, 0.611; 95\% CI, 0.486-3.416; $\mathrm{P}=0.611$ ), stroke (OR, 2.690; 95\% CI, 0.805-8.985; $\mathrm{P}=0.097)$, smoking $(\mathrm{OR}$, $1.543 ; 95 \% \mathrm{CI}, 0.659-3.611 ; \mathrm{P}=0.316)$ and alcohol consumption $(\mathrm{OR}, 1.110 ; 95 \% \mathrm{CI}, 0.454-2.716 ; \mathrm{P}=0.819)$ and severe stroke group (OR , 0.940; 95\% CI, 0.361-2.451; $\mathrm{P}=0.900$ ) were not found be significantly different between microemboli-positive and -negative patients following enrollment. In addition, no significant differences in age and total cholesterol, hematocrit, c-reactive protein and fibrinogen levels were identified (Table II).

Carotid artery ultrasound plaque, vascular symptomatic stenosis and the incidence of microemboli between various TOAST subtypes. The incidence of microemboli was found to vary between different etiologies and this difference was statistically significant. Patients with LA and cerebral embolism were found to be prone to microemboli. In addition, patients with symptomatic stenosis and unstable plaque were also found to be prone to development of microemboli (Table III).

Positive logistic regression analysis of microemboli. Logistic regression analysis of history of stroke, diabetes, coronary heart disease and antithrombotic therapy, low-density lipoprotein levels, TOAST grouping, artery stenosis, ultrasonic soft and hard plaque situation and results of the first microemboli tests were found to be significantly different depending on history of coronary heart disease and antithrombotic therapy and the occurrence rate of microemboli $(B=-1.341$, Wald $=39.886$, $\mathrm{P}=0.000$; Table IV).

Microemboli test of microemboli-positive patients 1 week following CI . Microemboli-positive patients (11/28) were identified as positive 1 week following CI, 9 were from the LA group (9/15), indicating that microemboli in patients with 
Table II. Etiology, stroke risk factors and general information of microemboli-positive and -negative patients.

\begin{tabular}{|c|c|c|c|c|}
\hline \multirow[b]{2}{*}{ Item } & \multirow[b]{2}{*}{ Patients (n) } & \multicolumn{2}{|c|}{ First determination of microemboli } & \multirow[b]{2}{*}{ P-value } \\
\hline & & Positive $(\mathrm{n}=28)$ & Negative $(n=107)$ & \\
\hline Gender male, n (\%) & 103 & $22(78.6)$ & $81(75.7)$ & 0.750 \\
\hline Average age, years & & $65.43 \pm 8.33$ & $64.86 \pm 10.89$ & 0.320 \\
\hline \multicolumn{5}{|l|}{ Patient history, n (\%) } \\
\hline Apoplexy & 13 & $5(17.9)$ & $8(7.5)$ & 0.097 \\
\hline Atrial fibrillation & 29 & $7(25.0)$ & $22(20.6)$ & 0.611 \\
\hline Hypertension & 68 & $17(60.7)$ & $51(47.7)$ & 0.219 \\
\hline Diabetes & 28 & $9(32.1)$ & $19(17.8)$ & 0.095 \\
\hline Coronary heart & 17 & $9(32.1)$ & $8(7.5)$ & 0.000 \\
\hline Alcohol consumption & 41 & $9(32.1)$ & $32(29.9)$ & 0.819 \\
\hline Smoking & 47 & $12(42.9)$ & $35(32.7)$ & 0.316 \\
\hline Antithrombotic therapy & 51 & $6(11.8)$ & $45(88.2)$ & 0.045 \\
\hline Hematocrit (\%) & & $38.83 \pm 5.03$ & $40.44 \pm 4.81$ & 0.836 \\
\hline Blood platelet count, $x 10^{9}$ & & $212.6 \pm 58.98$ & $214.1 \pm 63.46$ & 0.992 \\
\hline Fibrinogen, mg/l & & $2.968 \pm 0.59$ & $2.808 \pm 0.73$ & 0.359 \\
\hline c-reactive protein, mmol/l & & $3.36 \pm 3.69$ & $3.08 \pm 3.95$ & 0.359 \\
\hline Blood glucose, mmol/1 & & $6.83 \pm 3.04$ & $6.37 \pm 3.01$ & 0.704 \\
\hline Total cholesterol, mmol/l & & $4.45 \pm 0.99$ & $4.49 \pm 1.05$ & 0.585 \\
\hline Triglyceride, mmol/1 & & $1.76 \pm 1.17$ & $1.39 \pm 0.90$ & 0.137 \\
\hline Low density lipoprotein, mmol/1 & & $3.25 \pm 0.90$ & $2.77 \pm 0.82$ & 0.036 \\
\hline High density lipoprotein, $\mathrm{mmol} / \mathrm{l}$ & & $1.05 \pm 0.23$ & $1.11 \pm 0.25$ & 0.222 \\
\hline Apolipoprotein A, mmol/1 & & $1.14 \pm 0.23$ & $1.18 \pm 0.26$ & 0.696 \\
\hline Apolipoprotein B, mmol/l & & $0.98 \pm 0.28$ & $1.07 \pm 0.86$ & 0.414 \\
\hline Homocysteic acid, mmol/1 & & $15.42 \pm 8.75$ & $15.66 \pm 8.91$ & 0.937 \\
\hline Glutamyltranspeptidase, U/1 & & $35.65 \pm 13.23$ & $36.71 \pm 26.24$ & 0.140 \\
\hline Nerve function scores & & $5.32 \pm 3.95$ & $4.45 \pm 3.65$ & 0.781 \\
\hline $\begin{array}{l}\text { NIHSS score grouping } \\
\text { (mild-moderate), } \mathrm{n}(\%)\end{array}$ & 100 & $20(71.4)$ & $80(74.8)$ & 0.900 \\
\hline
\end{tabular}

main artery lesion types of CI are present for longer than microemboli of patients with other types of CI (Table V).

Correlation between microemboli and prognosis within 1 week and 3 months following CI. Neurological impairment NIHSS grading was performed immediately following $\mathrm{CI}$ and 1 week after admission. No significant difference in NIHSS grading was observed between microemboli-positive and -negative patients.

Patients who underwent stroke relapse within 1 week included 1 microemboli-positive patient with main artery lesions symptomatic stenosis, 1 microemboli-positive patient with cerebral embolism and 1 negative patient with aorta asymptomatic stenosis. The occurrence rate of stroke relapse in microemboli-positive and -negative patients was $7.1 \%(2 / 28)$ and $0.9 \%$ (1/107), respectively. Five patients underwent stroke relapse within 3 months following hospital discharge, including 3 cerebral embolism patients, 1 main artery lesion patient and 1 arteriolar lesion patient. The stoke recurrence rate between microemboli-positive and -negative patients was not found to be significantly different.
mRS grading of all patients was performed prior to hospital discharge. After 3 months, 89 outpatient follow-ups were scored directly and 31 patients underwent $\mathrm{mRS}$ grading by telephone follow-up. In these patients, there were 6 mortalities and 7 cases of recurrence during hospitalization and 3 months of follow-up, which indicated mRS aggravation. No significant difference between mRS grading and the initial microemboli-positive rate was identified. With regards to $\mathrm{CI}$ recurrence or the mortality of patients during the hospitalization period and within 3 months following hospital discharge (study endpoint event), a statistical significance was found between endpoint event and microemboli occurrence rate (Table VI).

\section{Discussion}

Microemboli are composed of blood clots, platelet aggregation particles and atherosclerotic plaque particles, (platelet, fibrinogen and cholesterol), as well as fat or air, and these abnormal materials are monitored by TCD ultrasound $(16,17)$. The presence of microemboli is indicative of unstable atherosclerotic plaques, which may lead to artery-to-artery 
Table III. TOAST grouping, carotid artery ultrasound plaque, vascular symptomatic stenosis and the incidence of microemboli.

\begin{tabular}{lccc}
\hline & & First determination of microemboli \\
\cline { 3 - 4 } Item & $\begin{array}{c}\text { Patients } \\
(\mathrm{n})\end{array}$ & $\begin{array}{c}\text { Positive, } \mathrm{n}(\%) \\
(\mathrm{n}=28)\end{array}$ & $\begin{array}{c}\text { Negative, } \mathrm{n}(\%) \\
(\mathrm{n}=107)\end{array}$ \\
\hline TOAST subgroups & & & $24(22.4)$ \\
Cerebral embolism & 33 & $9(32.1)$ & $34(31.8)$ \\
Aorta & 49 & $15(53.6)$ & $22(20.6)$ \\
Arteriole & 24 & $2(7.1)$ & $27(25.2)$ \\
Other & 29 & $2(7.1)$ & $84(86.6)$ \\
Arteria cervicalis ultrasound plaque & $13(13.4)$ & $15(75.0)$ \\
None & 97 & $5(25.0)$ & $8(44.4)$ \\
Stable & 20 & $10(55.6)$ & 0.000 \\
Unstable & 18 & $10(12.5)$ & $70(87.5)$ \\
Aorta stenosis & & & 0.011 \\
Non-stenosis & 80 & & \\
\hline
\end{tabular}

TOAST, Trial of Org 10172 in Acute Stroke Treatment.

Table IV. Positive logistic regression analysis of microemboli.

\begin{tabular}{|c|c|c|c|c|c|c|}
\hline Step & Parameters & $\mathrm{B}$ & SE & Wald & P-value & $\operatorname{Exp}(B)$ \\
\hline \multirow[t]{3}{*}{ 1(a) } & Coronary artery disease history & 1.658 & 0.575 & 8.326 & 0.004 & 5.249 \\
\hline & Antithrombotic therapy history & -1.373 & 0.553 & 6.159 & 0.013 & 0.253 \\
\hline & Constant & 0.228 & 0.603 & 0.143 & 0.705 & 1.256 \\
\hline
\end{tabular}

$\mathrm{B}$, coefficient of regression; SE, standard error; Wald, $\chi^{2}$ value; Exp, odds ratio.

Table V. Second microemboli test for microemboli-positive patients following 1 week.

\begin{tabular}{|c|c|c|c|c|}
\hline \multirow[b]{2}{*}{ Groups } & \multirow[b]{2}{*}{$\begin{array}{c}\text { Patients } \\
(n=28)\end{array}$} & \multicolumn{2}{|c|}{ Microemboli test, n (\%) } & \multirow[b]{2}{*}{ P-value } \\
\hline & & $\begin{array}{c}\text { Positive } \\
(\mathrm{n}=11)\end{array}$ & $\begin{array}{l}\text { Negative } \\
(n=17)\end{array}$ & \\
\hline Cerebral embolism & 9 & $2(18.2)$ & $7(41.2)$ & 0.050 \\
\hline Aorta & 15 & $9(81.8)$ & $6(35.3)$ & 0.050 \\
\hline Arteriole & 2 & 0 & $2(11.8)$ & 0.050 \\
\hline Other & 2 & 0 & $2(11.8)$ & 0.050 \\
\hline
\end{tabular}

embolism. Therefore, in recent years, a number of studies on microemboli monitoring in patients with ischemic stroke have been performed.

TOAST classification was initially proposed by Adams et al (18) in 1993 and has been revised over the last 20 years (19). The most widespread classification method involves the division of ischemic stroke into five types according to stroke etiology. In the present study, 135 stroke patients were analyzed. Among them, 33 patients (24.4\%) were classified with cardioembolism (cerebral embolism group), 49 (36.3\%) with atherosclerotic thrombotic CI (main artery group), 24 (17.8\%) with arteriolar lesions (arteriole group) and 29 (21.5\%) with other causes and unknown causes of stroke. Patients in the latter group were incorporated into one group for statistical purposes and named the stroke of other etiology group.

Following etiology grouping, statistical differences in history of atrial fibrillation, hypertension, diabetes and antithrombotic therapy were identified between the 4 subgroups. Blood glucose, total cholesterol and homocysteine levels were different between the subgroups; however, these differences were not found to be significant. In addition, no differences in age, gender, history of stroke, smoking and alcohol consumption, as well as blood lipid, fibrinogen and c-reactive protein levels were observed. 
Table VI. NIHSS score following 1 week.

\begin{tabular}{|c|c|c|c|c|}
\hline \multirow[b]{2}{*}{ Item } & \multirow[b]{2}{*}{$\begin{array}{l}\text { Patients } \\
(n=135)\end{array}$} & \multicolumn{2}{|c|}{ Initial microemboli status, n (\%) } & \multirow[b]{2}{*}{ P-value } \\
\hline & & $\begin{array}{c}\text { Positive } \\
(n=28)\end{array}$ & $\begin{array}{c}\text { Negative } \\
(n=107)\end{array}$ & \\
\hline \multicolumn{5}{|l|}{ NIHSS score } \\
\hline Improved & 65 & $13(26.4)$ & $52(28.6)$ & 0.345 \\
\hline No change & 52 & $9(32.1)$ & $43(40.2)$ & \\
\hline Aggravated & 18 & $6(21.4)$ & $12(11.2)$ & \\
\hline \multicolumn{5}{|l|}{$\mathrm{mRS}$ grading } \\
\hline Improved & 81 & $17(60.7)$ & $64(59.8)$ & 0.677 \\
\hline No change & 40 & $7(25.0)$ & $33(30.8)$ & \\
\hline Aggravated & 14 & $4(14.3)$ & $10(9.3)$ & \\
\hline \multicolumn{5}{|c|}{ Recurrent stroke or mortality } \\
\hline+ & 14 & $6(21.4)$ & $8(7.5)$ & 0.042 \\
\hline- & 121 & $22(78.6)$ & $99(92.5)$ & \\
\hline
\end{tabular}

mRS, modified Rankin score.

The occurrence rate of microemboli was different among the TOAST subtypes. The occurrence rate of microemboli was different among the TOAST subtypes. The microemboli occurrence rate of the main artery group was the highest, up to $30.6 \%$, and the micro-emboli occurrence rate of the cerebral embolism group was also high, up to $27.3 \%$. The microemboli occurrence rate of other groups was the lowest, only $6.9 \%$. The results indicated that patients with atherosclerotic thrombotic CI and cardiogenic cerebral embolism are more likely to develop microemboli than patients with arteriolar lesions, other causes or unknown causes. Stroke TOAST subtyping previously performed by Iguchi et al (12) reported no significant difference in the occurrence rate of microemboli between the subtypes.

Previous studies have revealed that patients with carotid artery atherosclerotic stenosis, intracranial main artery atherosclerosis stenosis and severe disorders of ventricular function, atrial fibrillation and valvular heart disease are at a significant risk of developing emboli and TCD testing is recommended in these cases (20). In addition, cerebral vascular angiography, interventional therapy of the nervous system may cause stripping resection of the carotid intima leading to arteriosclerotic plaque formation, with positive microemboli detection.

Comparison of carotid artery ultrasound plaque and symptomatic stenosis of patients and microemboli occurrence rate revealed that patients with unstable plaque symptomatic stenosis are more prone to developing microemboli. We hypothesize that this observation is associated with composition and properties of microemboli. The microemboli of patients with cardiogenic cerebral embolism are mainly composed of thrombus (21); however, the microemboli of atherosclerotic CI originates from arteriosclerotic unstable plaque rupture, which are platelet-rich particles $(22,23)$.

Microemboli-positive patients were reviewed 1 week following diagnosis and 11/28 cases remained positive, while
9/15 positive patients in the main artery group were negative following review (positive rate, 60\%). Significant differences in the number of patients that remained positive were found between the TOAST subgroups, indicating that the disappearance of microemboli in patients with main artery lesions is slower compared with other subgroups, and that unstable atherosclerotic plaques exhibit more plaque rupture, continuing to release microemboli.

Following the third microemboli test, 11 patients who were microemboli-positive in the second test were identified as microemboli-negative. Therefore, we hypothesized that the positive rate of microemboli was extremely low in the chronic and restoration phases of CI, having no clinical detection value. In this study, the overall incidence of microemboli in the 4 TOAST subgroups was $20.7 \%$ within $48 \mathrm{~h}$ following stroke. This decreased to $8.1 \% 1$ week following stroke. Microemboli-positive patients were retested and a rate of $39.3 \%$ was obtained, consistent with a previous study by Iguchi et al (12). Additional previous studies have demonstrated that the microemboli-positive rate immediately following stroke is highest and reduces over time $(7,24)$. Decreasing microemboli-positive rates are associated with natural history and antithrombotic and anticoagulant therapies (12).

In the present study, common risks and general clinical data were analyzed and significant differences with regard to first incidence of microemboli were identified in coronary heart disease, antithrombotic therapy and low-density lipoprotein levels (Table II). These factors were subjected to logistic regression analysis and a correlation between history of coronary heart disease and antithrombotic therapy and the occurrence rate of microemboli $(\mathrm{B}=-1.341 ; \mathrm{P}=0.000)$ was identified, indicating that coronary heart disease patients are prone to the development of microemboli, and that antithrombotic therapy may decrease the incidence of microemboli. Notably, a previous study reported that antiplatelet, anticoagulation and defibrase treatment decrease the positive rate of microemboli (25). 
NIHSS grading of the subgroups (mild-to-medium, grades 0-6 and severe, $>6$ ) was determined and the incidence of microemboli was found to be significantly different between the subgroups, consistent with previous studies $(26,27)$, indicating that the incidence of microemboli is not associated with neurological deficit severity. However, the present study revealed that there was no correlation between the existence of microemboli and clinical symptomatic recurrent stroke within 7 days following stroke, but the microemboli lead to small infarct lesions which are not observed by MRI DWI, indicative of a decrease in asymptomatic cerebral perfusion $(28,29)$. mRS grading prior to hospital discharge and 3 months following $\mathrm{CI}$ was compared with the first positive rate of microemboli. Results were not found to be significantly different, indicating that development of microemboli and improvement of neurological deficits are not correlated.

Iguchi et al $(12,30)$ reported that it is within $48 \mathrm{~h}$, not $24 \mathrm{~h}$, following stroke that microemboli presence is associated with recurrence of stroke. Therefore, in the current study, the first microemboli test was performed $48 \mathrm{~h}$ after stroke. Relapse within 1 week was observed in 3 patients, 2 of which were main artery lesions symptomatic stenosis microemboli-positive patients and 1 was a cerebral embolism microemboli-negative patient. The recurrence rate of microemboli-positive patients $(7.4 \% ; 2 / 28)$ was higher than that of microemboli-negative patients $(0.9 \% ; 1 / 107)$; however, this difference was not statistically significant. This may be explained by the small sample size and low recurrent rate of stroke in this study. We hypothesize that the rupture of large artery stenosis plaques and sustained release of microemboli are the main causes of recurrent stroke in patients with large artery lesions. In this study, the overall detection rate of recurrent stroke was comparatively low. The reason may be that, as there were no clear clinical symptoms of recurrent stroke, reexamination with MRI DWI was not performed. Previously, Kang et al (31) revealed that small new infarcts may occur in $34 \%$ patients with no clinical symptoms and no marked changes in NIHSS grading.

CI recurrence (7 cases) and mortality directly resulting from CI (6 cases) was considered the endpoint of the study. A significantly higher number of microemboli-positive patients were found to reach endpoint compared with microemboli-negative patients within $48 \mathrm{~h}$ following CI, indicating that microemboli-positive patients are more prone to stroke recurrence and mortality resulting from stroke, consistent with previous studies (28). Due to the small sample size, the number of stroke recurrences in microemboli-positive patients (10.7\%) was higher than microemboli-negative patients (3.7\%), but was not found to be statistically significant. Previous studies have reported that the existence of microemboli is associated with stroke recurrence (5,10-12).

In summary, results of the present study indicate that the incidence of microemboli in various etiologically classified TOAST subtypes is significantly different, and that stroke recurrence and mortality rates in microemboli-positive patients 3 months following CI were increased compared with microemboli-negative patients. Therefore, microemboli testing is useful for analysis of stoke etiology and the prediction of stroke recurrence and mortality. The single stroke recurrence rate of microemboli-positive patients was higher than that of microemboli-negative patients, but this difference was not found to be significant. Further studies require a larger sample size and a longer follow-up time. In this study, the time of microemboli detection was only $30 \mathrm{~min}$, which may decrease the detection rate of microemboli.

\section{Acknowledgements}

The current study was supported by the Lianyungang Municipal Health Bureau Fund (no. ZC107).

\section{References}

1. Spencer MP, Thomas GI, Nicholls SC, et al: Detection of middle cerebral artery emboli during carotid endarterectomy using transcranial Doppler ultrasonography. Stroke 21: 415-423, 1990.

2. No authors listed: Basic identification criteria of Doppler microembolic signals. Consensus Committee of the Ninth International Cerebral Hemodynamic Symposium. Stroke 26: 1123, 1995.

3. Grosset DG, Georgiadis D, Kelman AW, et al: Detection of microemboli by transcranial Doppler ultrasound. Tex Heart Inst J 23: 289-292, 1996.

4. Al-Mubarak N, Roubin GS, Vitek JJ, et al: Effect of the distal-balloon protection system on microembolization during carotid stenting. Circulation 104: 1999-2002, 2003.

5. Droste DW, Junker K, Hansberg T, et al: Circulating microemboli in 33 patients with intracranial arterial stenosis. Cerebrovasc Dis 13: 26-30, 2002.

6. Gao S, Wong KS, Hansberg T, et al: Microembolic signal predicts recurrent cerebral ischemic events in acute stroke patients with middle cerebral artery stenosis. Stroke 35: 2832-2836, 2004.

7. Sliwka U, Lingnau A, Stohlmann WD, et al: Prevalence and time course of microembolic signals in patients with acute stroke. A prospective study. Stroke 28: 358-363, 1997.

8. Wong KS, Gao S, Chan YL, et al: Mechanisms of acute cerebral infarctions in patients with middle cerebral artery stenosis: a diffusion-weighted imaging and microemboli monitoring study. Ann Neurol 52: 74-81, 2002.

9. Kimura K, Minematsu K, Koga M, et al: Microembolic signals and diffusion-weighted MR imaging abnormalities in acute ischemic stroke. AJNR Am J Neuroradiol 22: 1037-1042, 2001.

10. Nakajima M, Kimura K, Shimode A, et al: Microembolic signals within 24 hours of stroke onset and diffusion-weighted MRI abnormalities. Cerebrovasc Dis 23: 282-288, 2007.

11. Markus HS and MacKinnon A: Asymptomatic embolization detected by Doppler ultrasound predicts stroke risk in symptomatic carotid artery stenosis. Stroke 36: 971-975, 2005.

12. Iguchi Y, Kimura K, Kobayashi K, Yamashita S, Shibazaki K and Inoue T: Microembolic signals after 7 days but not within 24 hours of stroke onset should be predictor of stroke recurrence. J Neurol Sci 263: 54-58, 2007.

13. Valton L, Larrue V, le Traon AP, et al: Microembolic signals and risk of early recurrence in patients with stroke or transient ischemic attack. Stroke 29: 2125-2128, 1998.

14. DeGraba TJ, Hallenbeck JM, Pettigrew KD, et al: Progression in acute stroke: value of the initial NIH stroke scale score on patient stratification in future trials. Stroke 30: 1208-1212, 1999.

15. Hao WH, Leung KS, Wong, et al: The significance of microembolic signals and new cerebral infarcts on the progression of neurological deficit in acute stroke patients with large artery stenosis. Cerebrovasc Dis 29: 424-430, 2010.

16. Markus HS and Brown MM: Differentiation between different pathological cerebral embolic materials using transcranial Doppler in an in vitro model. Stroke 24: 1-5, 1993.

17. Junghans U and Siebler M: Cerebral microembolism is blocked by tirofiban, a selective nonpeptide platelet glycoprotein IIb/IIIa receptor antagonist. Circulation 107: 2717-2721, 2003.

18. Adams HP, Bendixen BH, Kappelle LJ, et al: Classification of subtype of acute ischemic stroke. Definitions for use in a multicenter clinical trial. TOAST. Trial of Org 10172 in Acute Stroke Treatment. Stroke 24: 35-41, 1993.

19. Goldstein LB, Jones MR, Matchar DB, et al: Improving the reliability of stroke subgroup classification using the Trial of ORG 10172 in Acute Stroke Treatment (TOAST) criteria. Stroke 32: 1091-1098, 2001. 
20. Nabavi DG, Arato S, Droste DW, et al: Microembolic load in asymptomatic patients with cardiac aneurysm, severe ventricular dysfunction and atrial fibrillation. Clinical and hemorheological correlates. Cerebrovasc Dis 8: 214-221, 1998.

21. Kumral E, Balkir K, Uzuner N, et al: Microembolic signal detection in patients with symptomatic and asymptomatic lone atrial fibrillation. Cerebrovasc Dis 12: 192-196, 2001.

22. Goertler M, Blaser T, Krueger S, et al: Cessation of embolic signals after antithrombotic prevention is related to reduced risk of recurrent arterioembolic transient ischaemic attack and stroke. J Neurol Neurosurg Psychiatry 72: 338-342, 2002.

23. Junghans U and Siebler M: Cerebral microembolism is blocked by tirofiban, a selective nonpeptide platelet glycoprotein IIb/IIIa receptor antagonist. Circulation 107: 2717-2721, 2003.

24. Delcker A, Schnell A and Wilhelm H: Microembolic signals and clinical outcome in patients with acute stroke - a prospective study. Eur Arch Psychiatry Clin Neurosci 250: 1-5, 2000.

25. Goertler M, Baeumer M, Kross R, et al: Rapid decline of cerebral microemboli of arterial origin after intravenous acetylsalicylic acid. Stroke 30: 66-69, 1999.

26. Lund C, Rygh J, Stensrød B, et al: Cerebral microembolus detection in an unselected acute ischemic stroke population. Cerebrovasc Dis 10: 403-408, 2000
27. Abbott AL, Chambers BR, Stork JL, et al: Embolic signals and prediction of ipsilateral stroke or transient ischemic attack in asymptomatic carotid stenosis: a multicenter prospective cohort study. Stroke 36: 1128-1133, 2005

28. Iosif C, Oppenheim C, Lamy C, et al: Bilateral hypoperfusion and normal diffusion MR images in a case of acute embolic stroke. Cerebrovasc Dis 26: 95-96, 2008.

29. Schlaug G, Benfield A, Baird AE, et al: The ischemic penumbra: operationally defined by diffusion and perfusion MRI. Neurology 53: 1528-1537, 1999.

30. Iguchi Y, Kimura K, Kobayashi K, et al: Microembolic signals at 48 hours after stroke onset contribute to new ischaemia within a week. J Neurol Neurosurg Psychiatry 79: 253-259, 2008.

31. Kang DW, Kwon SU, Yoo SH, et al: Early recurrent ischemic lesions on diffusion-weighted imaging in symptomatic intracranial atherosclerosis. Arch Neurol 64: 50-54, 2007. 